\title{
Valoración de las dimensiones del paladar y su relación con alteraciones verticales.
}

\author{
Daniel Maravillas García, ${ }^{1}$ \\ Tatiana Dinhora Mondragón Báez, ${ }^{2}$ \\ Abraham Mendoza Quintanilla, ${ }^{3}$ \\ Rogelio Danovan Venegas Lancón. ${ }^{4}$
}

Resumen

Objetivos: La presente investigación tuvo como objetivo establecer la correlación entre la presencia de maloclusiones a nivel vertical con el índice del paladar y con la altura palatina. Materiales y Métodos: Estudio de tipo transversal. La población de estudio fue conformada por 134 modelos de yeso con dentición mixta, 81 mujeres y 53 hombres, seleccionados por conveniencia y obtenidos desde el 2014 hasta el 2018 del área de clínica de odontopediatría y ortodoncia en la Escuela Nacional de Estudios Superiores Unidad León. La muestra de estudio fue dividida en 3 categorías según la oclusión; mordida abierta (37), mordida profunda (32) y normoclusión (65). Se realizaron mediciones del ancho intermolar y altura del paladar con el uso de un calibrador digital, posteriormente se obtuvo el índice del paladar y se correlacionó con los parámetros expuestos por Korkhaus mediante la prueba estadística de Spearman. Resultados: La prueba estadística de Spearman refleja que no hay correlación entre la magnitud de los valores del índice del paladar propuestos por Korkhaus con la presencia de maloclusiones a nivel vertical. De igual manera las pruebas estadísticas indican que no existe correlación notable entre la altura del paladar y la oclusión en relación vertical. Conclusiones: El resultado obtenido al usar el índice del paladar no fue capaz de corresponder a los parámetros propuestos por Korkhaus como normales, posiblemente al ser usado durante la etapa de la dentición mixta pues durante este periodo suceden cambios constantes relacionados al crecimiento.

Palabras clave: Mordida abierta, Hábitos orales, Dentición mixta.

\footnotetext{
${ }^{1}$ Odontólogo, Guanajuato, México.

${ }^{2}$ Maestra y coordinadora del Área de Profundización en Odontopediatría y Posgrado en Odontología Pediátrica, UNAM ENES, León, Guanajuato, México. ${ }^{3}$ Odontopediatria, Docente del Área de Profundización en Odontopediatría y Posgrado en Odontología Pediátrica, UNAM ENES-León, Guanajuato, México.

${ }^{4}$ Maestro, Docente y coordinador del laboratorio de Prótesis Dental, Guanajuato, México.
} 
Artigo original

\section{Avaliação das dimensões do palato e sua relação com alterações verticais.}

\section{Resumo}

Objetivos: Os objetivos a presente investigação buscou valor uma correlação entre a presença de más oclusões em nível vertical com o índice do palato e com a altura palatina. Materiais e Métodos: Estudo do tipo transversal. A população do estudo foi composta por 134 modelos de gesso com dentição mista, 81 mulheres e 53 homens, selecionados por conveniência e obtidos de 2014 a 2018 na área de Odontopediatria e Ortodontia na Escola Nacional de Ensino Superior Unidade de León. A amostra do estudo foi dividida em 3 categorias de acordo com a oclusão; mordida aberta (37), mordida profunda (32) e normoclusão (65). A medida da largura intermolar e da altura do palato foi feita com o uso de um paquímetro digital, o índice de palato foi posteriormente obtido e relacionado aos parâmetros expostos por Korkhaus por meio do teste estatístico de Spearman. Resultados: $\mathrm{O}$ teste estatístico deSpearman mostra que não há correlação entre a magnitude dos valores do índice de palato proposta por Korkhaus e a presença de más oclusões verticais. Da mesma forma, os testes estatísticos indicam que não há correlação notável entre a altura do palato e a oclusão na relação vertical. Conclusões: $\mathrm{O}$ resultado obtido com o uso do índice de palato não foi capaz de corresponder aos parâmetros propostos por Korkhaus como normais, possivelmente quando usado durante o estágio de dentição mista, porque durante este período há mudanças constantes relacionadas ao crescimento.

Palavras chaves: Mordida aberta, Hábitos bucais, Dentição mista.

Original article

\section{Assessment of palate dimensions and its relation with vertical alterations}

\begin{abstract}
Purpose: The aim of the present study is to assess the relation between vertical malocclusions with palatal index and palatal height. Materials and Methods: Transversal study. The sample consisted in 134 stone models within mixed dentition, 81 females and 53 males, they were convenience selected and obtained from the pediatric and orthodontic dentistry medical center at the National School for Superior Studies Unit León
\end{abstract}

since 2014 to 2018. The stone models were divided into three categories according to their kind of occlusion: open bite (37), deep bite (32) and normal bite (65). Palatal width and height were measure by using a digital caliper. Subsequently the palatal index was obtained and it was related with the parameters exposed by Korkhaus using the Spearman statistical test. Results: The Spearman statistical test indicated there is no a clearly relationship between palatal index values exposed by Korkhaus with the presence 
of vertical malocclusions and in the same way there is no a relationship between palatal height and vertical malocclusions. Conclusions: The result obtained by using the palatal index was no able to indicate an agreement with Korkhaus parameters.

\section{Introducción}

La anatomía del paladar tiene un rango de variaciones anatómicas tanto en estructura, forma y tamaño, por lo que los antropólogos han relacionado su desarrollo dentro del maxilar a factores como la dieta, el medio ambiente y condiciones genéticas entre las diferentes poblaciones. El paladar se ha constituido como una pieza clave para el desarrollo del patrón esqueletal debido a su posición en el cráneo. Las investigaciones clínicas relacionadas a la altura del paladar lo han descrito con términos subjetivos como alto, angosto, poco profundo o normal, en donde el único criterio que existe para considerarlo anormal es cuando la altura del paladar es el doble de la altura de los dientes. La altura del paladar se ha empleado como método de diagnóstico en síndromes que conllevan alguna alteración en el complejo facial, por ejemplo: paladar hendido, motivo por el cual los estudios relativos a la medición de su altura se han concentrado a síndromes craneofaciales y dientes mal posicionados. ${ }^{1,2}$

Durante el periodo de crecimiento la posición de la lengua participa activamente en el desarrollo de las estructuras esqueletales. Una bóveda palatina profunda se ha asociado a una postura baja de la lengua, ya que produce un descenso en la presión de la boca al cierre
Probably this disagreement was due to the constant changes that occur during the period of the mixed dentition.

Key words: Open bite, Oral habits, Mixed dentition.

provocando un estímulo a los procesos alveolares del maxilar y por consiguiente un aumento en la altura del paladar. Caso contrario una postura más alta de la lengua se asocia con la activación de los músculos de cierre de la mandíbula la cual somete a los procesos alveolares creando una presión ascendente, reduciendo la altura del paladar, dicho en pocas palabras, la bóveda palatina se puede ajustar durante la etapa de crecimiento en función con la postura de la lengua. ${ }^{3}$

Durante la transición de la dentición primaria a la permanente, la forma y ancho del arco dental se ve en constante cambio debido al crecimiento vertical de los procesos alveolares que ocurren durante el movimiento dental, la magnitud de estos cambios puede ser influenciada por alteraciones presentes en el ambiente. La presencia de hábitos orales repercute en el equilibrio de las estructuras faciales. Popovich y Thompson ${ }^{5}$ en 1983, sugirieron que existe una relación entre la prevalencia de maloclusiones con algún tipo de hábito y Ovsenik ${ }^{6}$ en 2007, consideró que la presencia de hábitos como la succión, tienen un impacto indirecto en el cambio de postura de la deglución sin importar el tiempo de duración que haya estado presente el hábito. Yemitan ${ }^{7}$ en 2010, propuso que existe una relación entre la succión digital con la disminución del ancho del maxilar, así como un aumento en 
la profundidad del paladar y reducción de la distancia intercanina en la mandíbula. ${ }^{4-7}$

Para obtener un equilibrio en el desarrollo normal del complejo facial depende de un proceso multifuncional que se ve afectado por factores genéticos y ambientales. La respiración, succión, masticación, deglución y fonación son partes del sistema neuromuscular que representan el control natural para un adecuado estímulo, desarrollo y crecimiento de las estructuras faciales en donde, cualquier alteración negativa trae como consecuencia anormalidades que pueden afectar a las bases óseas. De acuerdo a la teoría de la matriz funcional de Moss menciona que la actividad de la respiración nasal influencia y favorece el desarrollo de las estructuras craneofaciales (mandíbula, arcos dentales, maxilar, lengua y músculos faciales) debido a la adecuada interacción con la masticación y deglución. ${ }^{8-10}$

En el momento de la inspiración la lengua se eleva y se ve proyectada contra el paladar, ejerciéndose un estímulo positivo para su desarrollo. Cuando el infante comienza a respirar por la boca, la lengua adopta una posición descendente para permitir el paso del flujo del aire y se dejan de excitar las terminaciones neuronales que coordinan el crecimiento iniciando un proceso de adaptación funcional que influye en la relación músculo-esquelético así como se crea un desbalance entre las matrices funcionales responsables de la transformación de tamaño y forma. Cuando la respiración se realiza a través de la boca se producen una serie de efectos secundarios capaces de producir un efecto nocivo en los maxilares y sus músculos. La literatura científica indica que la influencia de la respiración oral sobre el rostro induce un patrón respiratorio que incrementa la presión en la cavidad oral y en la cavidad nasal. Cualquier cambio en el equilibrio se debe a la posición baja de la mandíbula, induciendo a los músculos buccinadores a causar una presión lateral en el arco maxilar, lo cual estrecha el arco y resulta en un incremento en la profundidad del paladar. ${ }^{10-13}$

Una bóveda palatina profunda y estrecha es una característica distintiva de los pacientes con mordida abierta; la cual se ha relacionado como uno de los efectos secundarios a la obstrucción nasal, la profundidad de la bóveda palatina también se puede interpretar como el resultado de un sobrecrecimiento de la altura alveolar posterior del maxilar. Los sujetos con maloclusión de mordida abierta, tienden a mostrar un paladar angosto, una deglución atípica y una posición de la lengua más adelantada durante el reposo. Un paladar angosto o triangular es efecto de una presión provocada por la lengua sobre los dientes inferiores mientras se aumenta el ancho del arco mandibular y provoca el colapso del arco maxilar. ${ }^{14,15}$

Korkhaus en 1939, instauró un parámetro de normalidad de $42 \%$ al utilizar el índice del paladar, el cual define la altura del paladar como la vertical al plano del rafé medio el porcentaje aumenta en caso de mayor elevación del paladar con respecto al diámetro transversal y disminuye cuando el paladar se aplana. El paladar se eleva como síntoma fundamental de una compresión apical de las apófisis alveolares, anomalía presente en sujetos con una respiración bucal crónica y en determinados hábitos de succión digital. Si el valor disminuye puede ser indicio de una sobre expansión del maxilar o un gran descenso del paladar. ${ }^{16}$ 
La presente investigación tuvo como objetivo valorar la correlación entre la presencia de maloclusiones a nivel vertical con el índice del paladar obtenido a través de la medición de las dimensiones de anchura y altura del paladar y relacionar la altura del paladar con maloclusiones verticales en modelos de yeso con dentición mixta.

\section{Materiales y métodos}

La investigación se realizó en la clínica de odontopediatría y ortodoncia de la Escuela Nacional de Estudios Superiores Unidad León bajo la modalidad de campo de estudio transversal. La población de estudio fue conformada por 134 modelos de yeso con dentición mixta obtenidos desde el 2014 hasta el 2018 los cuales fueron seleccionados por conveniencia, los modelos de estudio fueron tomados como método auxiliar de diagnóstico clínico, por lo que los padres y/o tutores de los pacientes firmaron el consentimiento informado para poder utilizar información y material obtenido con fines académicos y/o investigación.

La muestra estuvo conformada por 53 modelos de yeso de hombres y 81 modelos de yeso de mujeres, se dividieron en 3 categorías: mordida abierta (37), mordida profunda (32) y normoclusión (65).

Para obtener las dimensiones del paladar se tomaron en cuenta los siguientes puntos de referencia:

Ancho intermolar: intersección a nivel mesial de los primeros molares permanentes.
Altura del paladar: base a nivel del rafe medio con intersección a mesial de los primeros molares permanentes y su vértice a nivel del plano oclusal.

Para la medición del ancho intermolar se utilizó un calibrador digital modelo HER411 marca STEREN® el cual se colocó en los puntos de referencia previamente expuestos y para conseguir la altura del paladar se colocó una barra paralela al nivel oclusal y con el calibrador digital se obtuvieron las mediciones correspondientes en los modelos de todos los grupos (Figura 1 y 2).

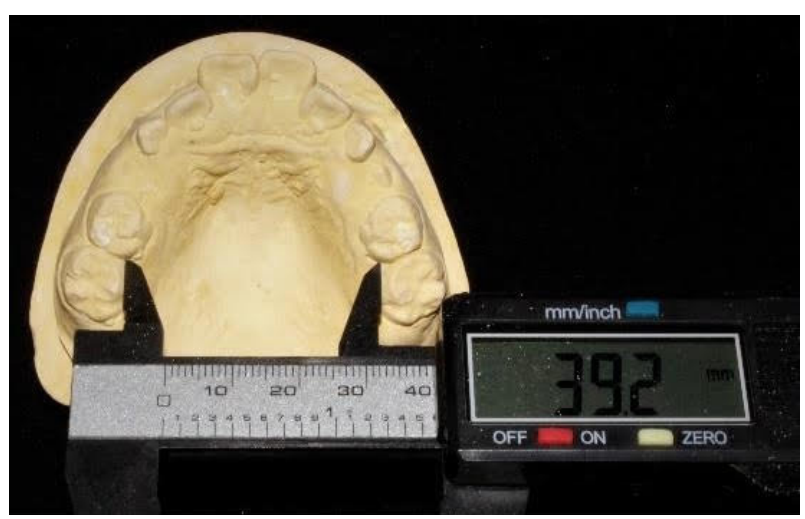

Figura 1: Medida del ancho intermolar.

Fuente: Directa

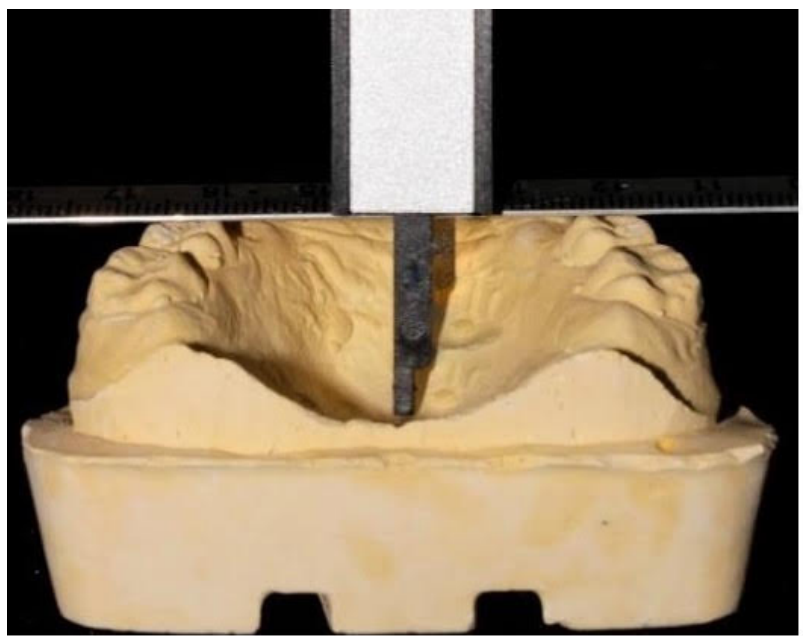

Figura 2: Altura del paladar

Fuente: Directa 
Fue utilizado el índice del paladar el cual fue descrito por Korkhaus en 1939 y se obtiene mediante la siguiente fórmula:

$$
\frac{\text { Altura del paladar }}{\text { Ancho posterior de la arcada }} \times 100=
$$

$>42 \%=$ falta de desarrollo transversal $\mathrm{y} / \mathrm{o}$ falta de descenso del paladar.

42\% Valor promedio normal.

$<42 \%$ = sobreexpansión y/o gran descenso del paladar

Se realizaron las mediciones de los modelos con un solo observador, para la calibración se realizó una primera medición del ancho intermolar y la altura del paladar en los
134 modelos y después de una semana se realizó una segunda medición a 23 modelos de estudio escogidos al azar, los datos de ambas ocasiones fueron analizados con la prueba de coeficiente Kappa de Cohen mediante el programa estadístico SPSS Statistics ${ }^{\circledR}$ indicando un valor de 0.42 que es considerado como aceptable (Tabla 1).

Posteriormente se realizó la prueba de normalidad Shapiro Wilk para contrastar la normalidad de los datos obtenidos. Una vez realizadas las pruebas de normalidad se realizaron pruebas de correlación no paramétrica de Spearman para corroborar si existe algún tipo de correlación entre índice del paladar con la oclusión en relación vertical y la altura del paladar con la oclusión en relación vertical (Tabla 2).

Tabla 1. Resultados prueba Coeficiente de Kappa.

\begin{tabular}{|l|c|c|c|c|}
\hline & Valor & $\begin{array}{c}\text { Error estándar } \\
\text { asintótico }^{a}\end{array}$ & T aproximadab $^{b}$ & $\begin{array}{c}\text { Significación } \\
\text { aproximada }\end{array}$ \\
\hline Medida de acuerdo Kappa & 0.042 & 0.039 & 4.796 & 0.000 \\
\hline N de casos válidos & 23 & & & \\
\hline
\end{tabular}

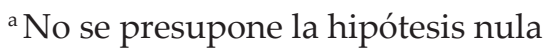

${ }^{\mathrm{b}}$ Utilización del error estándar asintótico que presupone la hipótesis nula

Tabla 2. Resultados prueba de normalidad Shapiro-Wilk

\begin{tabular}{|l|l|l|l|l|l|c|c|}
\hline \multirow{2}{*}{$\begin{array}{c}\text { Oclusión en relación } \\
\text { vertical }\end{array}$} & \multicolumn{2}{|c|}{\begin{tabular}{c} 
Kolmogorov-Smirnova \\
\cline { 3 - 9 }
\end{tabular}} & Estadístico & gl & Sig. & \multicolumn{3}{|c|}{ Estadístico } & gl & Sig. \\
\hline \multirow{3}{*}{$\begin{array}{l}\text { Índice del paladaro-Wilk } \\
\text { (Korkhaus) }\end{array}$} & Mordida abierta & 0.111 & 37 & $0.200^{*}$ & 0.961 & 37 & 0.212 \\
\cline { 2 - 10 } & Normoclusión & 0.079 & 65 & $0.200^{*}$ & 0.976 & 65 & 0.240 \\
\cline { 2 - 10 } & Mordida profunda & 0.101 & 32 & $0.200^{*}$ & 0.972 & 32 & 0.549 \\
\hline
\end{tabular}

* Esto es un límite inferior de la significación verdadera

${ }^{a}$ Corrección de significación de Lilliefors 


\section{Resultados}

Los resultados referentes al índice del paladar muestran una media mayor a la propuesta como normal por Korkhaus $(42 \%)$ teniendo el grupo con mordida abierta una media de $46.1 \%$, el grupo con mordida profunda un $44.2 \%$ y el grupo con normoclusión un $45.3 \%$. A su vez la información obtenida por la prueba estadística de Spearman sobre la correlación entre la oclusión en relación vertical / índice del paladar (Korkhaus) indica que no hay correlación entre ambas variables, es decir la magnitud de los valores del índice del paladar propuestos por Korkhaus no se relaciona proporcionalmente con la presencia de maloclusiones a nivel vertical (Tabla 3 ).

Los resultados de la correlación entre la oclusión en relación vertical/altura del paladar de acuerdo a los datos obtenidos la media total de la altura del paladar fue de $17.2 \mathrm{~mm}$, la media en la categoría de mordida abierta fue de $17.1 \mathrm{~mm}$, en la normoclusión fue $17.2 \mathrm{~mm}$ y en la mordida profunda fue $16.8 \mathrm{~mm}$ (Tabla 4). La información proporcionada por la prueba estadística de Spearman revela que no existe correlación relevante entre las variables de la altura del paladar y la oclusión en relación vertical (Tabla 5).

Tabla 3. Correlación no paramétrica entre la oclusión en relación vertical y el índice del paladar.

\begin{tabular}{|c|c|c|c|c|}
\hline \multicolumn{3}{|c|}{ Correlaciones } & $\begin{array}{l}\text { Oclusión en } \\
\text { relación vertical }\end{array}$ & $\begin{array}{l}\text { Índice del paladar } \\
\text { (Korkhaus) }\end{array}$ \\
\hline \multirow{6}{*}{$\begin{array}{l}\text { Rho de } \\
\text { Spearman }\end{array}$} & \multirow{3}{*}{$\begin{array}{l}\text { Oclusión } \\
\text { en relación } \\
\text { vertical }\end{array}$} & Coeficiente de correlación & 1.000 & -.099 \\
\hline & & Sig. (bilateral) & $\cdot$ & .254 \\
\hline & & $\mathrm{N}$ & 134 & 134 \\
\hline & \multirow{3}{*}{$\begin{array}{l}\text { Índice del } \\
\text { paladar } \\
\text { (Korkhaus) }\end{array}$} & Coeficiente de correlación & -.099 & 1.000 \\
\hline & & Sig. (bilateral) & .254 & . \\
\hline & & $\mathrm{N}$ & 134 & 134 \\
\hline
\end{tabular}

Tabla 4. Altura promedio del paladar

\begin{tabular}{|l|l|}
\hline Media Total & $17.2 \mathrm{~mm}$ \\
\hline Media Mordida abierta & $17.1 \mathrm{~mm}$ \\
\hline Media Normoclusión & $17.2 \mathrm{~mm}$ \\
\hline Media Mordida profunda & $16.8 \mathrm{~mm}$ \\
\hline
\end{tabular}

Tabla 5. Correlación no paramétrica entre la oclusión en relación vertical y la altura del paladar.

\begin{tabular}{|l|l|l|c|c|}
\hline \multicolumn{2}{|c|}{ Correlaciones } & $\begin{array}{c}\text { Oclusión en } \\
\text { relación vertical }\end{array}$ & $\begin{array}{c}\text { Altura del paladar } \\
(\mathrm{mm})\end{array}$ \\
\hline \multirow{3}{*}{\begin{tabular}{l}
\multirow{4}{*}{$\begin{array}{l}\text { Oclusión } \\
\text { Rho de } \\
\text { en relación } \\
\text { vertical }\end{array}$} \\
\cline { 2 - 5 }
\end{tabular}} & Coeficiente de correlación & 1.000 & -.032 \\
\cline { 2 - 5 } & Sig. (bilateral) & $\mathrm{N}$ & 134 & .709 \\
\cline { 2 - 5 } & $\begin{array}{l}\text { Altura del } \\
\text { paladar } \\
(\mathrm{mm})\end{array}$ & Coeficiente de correlación & -.032 & 134 \\
\cline { 3 - 5 } & Sig. (bilateral) & .709 & 1.000 \\
\cline { 2 - 5 } & $\mathrm{N}$ & 134 &. \\
\hline
\end{tabular}




\section{Discusión}

En investigaciones previas se ha encontrado que el índice del paladar es frecuentemente superior con lo determinado por Korkhaus. Este estudio tiene similitudes con los resultados encontrados por otros autores como Younes et $\mathrm{al}^{1}$ que, en 1995, determinaron que el índice del paladar estudiado en sujetos con normoclusión y dentición mixta fue mayor a lo que Korkhaus había determinado como normal. De mismo modo Biling Spring ${ }^{14}$ en 1998 y Shruti ${ }^{17}$ en 2012, determinaron que la altura del paladar no guarda relación con la presencia de una mordida abierta pues puede presentarse una altura evidente en cualquier dentición y tipo de oclusión.

Otros autores como El-Badry et al ${ }^{2}$ en 2008, señalan que el ancho intermolar incrementa como respuesta a la edad y que la altura del paladar no representa una diferencia significativa durante la transición de la dentición mixta y Susan Howell ${ }^{18}$ en 1981, menciona en sus estudios que hay un pequeño incremento de la altura del paladar desde la dentición primaria a la mixta y de ésta a la permanente y que las variaciones encontradas en los diversos estudios sobre la altura y ancho del paladar puede ser conectadas al uso de diferentes instrumentos y puntos de referencia escogidos.

Es así que los resultados encontrados en este estudio tienen relación con lo encontrado por los autores previamente mencionados, sin embargo la forma de realizar las mediciones, los instrumentos utilizados y los grupos que fueron seleccionados juegan un papel muy importante al momento de comparar los resultados expuestos, pues a pesar de ser muy similares los estudios ninguno pretendió buscar cual es la relación existente entre las dimensiones del paladar o su índice con la presencia de alteraciones que pudieran afectar el plano vertical.

\section{Conclusiones}

De acuerdo a los resultados obtenidos la media total de la altura del paladar fue de $17.2 \mathrm{~mm}$, la media en la categoría de mordida abierta fue de $17.1 \mathrm{~mm}$, en la normoclusión fue $17.2 \mathrm{~mm}$ y en la mordida profunda fue $16.8 \mathrm{~mm}$. En las pruebas estadísticas de Spearman revelan que no existe correlación entre la altura del paladar y la oclusión en relación vertical.

\section{Referencias bibliográficas}

1. Younes S, Angbawi M, Dosari A. A comparative study of palatal height in a Saudi and Egyptian population. J Oral Rehabil. 1995;22:391-395.

2. El-Badry, H., Nemat, H., Mostafa, I, et al. Palatal dimensions in Egyptian children at the mixed dentition period. Int. J Dent. Anthropol 2008;12:15-20.

3. Bourdiol P, Mishellany-Dutour A, Abou-El-Karam S, et al. Is the tongue position influenced by the palatal vault dimensions? J Oral Rehabil 2010;37:100-106.

4. Amirabadi GE, Golshah A, Derakhshan S, et al. Palatal dimensions at different stages of dentition in 5 to 18 year old Iranian children and adolescent with normal occlusion. BMC Oral Health 2018;18;1-6.

5. Popovich F, Thompson FG. Thumb and fingersucking; its relation to malocclusion. Am J Orthod 1983; 63(5): 148-155. 
6. Ovsenik M, Marjan F, Korpar $\mathrm{M}$ et al. Follow-up study of functional and morphological malocclusion trait changes from 3 to 12 years of age. European Journal of Orthodontics 2007; 29(5):523-529

7. Yemitan TA, daCosta OO, Sanu OO et al. Effects of digit sucking on dental arch dimensions in the primary dentition. Afr J Med Med Sci. 2010 Mar;39(1):55-61

8. Montaldo L, Montaldo P, Cuccaro, et al. Effects of feeding on non-nutritive sucking habits and implications on occlusion in mixed dentition. Int. J Paediatr. Dent. 2011;21:68.73.

9. Fatima D. Evaluation of the palate dimensions of patients with perennial allergic rhinitis. Int. J. Paediatr. Dent. 2001;11:365-371.

10. Harari D. The Effect of Mouth Breathing Versus Nasal Breathing on Dentofacial and Craniofacial Development in Orthodontic Patients. The Laryngoscope 2010;120: 2089-2093.

11. Zoila V, Lianet F. Anay D. Repercusión de la respiración bucal en el sistema estomatognático en niños de 9 a 12 años. Rev Ciencias Médicas 2013;17:126-137.

12. McNamara JR, James A. Influence of Respiratory Pattern On Craniofacial Growth. The Angle Orthod 1981;51:269-300.

13. Gail S, Peter S. Nasal Airway Obstruction and Facial Development. J. Allergy Clin. Immunol 1984;2:225235.

14. Biling S. The nature of arch width difference and palatal depth of the anterior open bite. Am J Orthod Dentofacial Orthop 1998;113:344-350.

15. Louly F, Nouer PA, Janson G, et al. Dental arch dimensions in the mixed dentition: a study of Brazilian children from 9 to 12 years of age. J. Appl. Oral Sci 2011;19:169:174.

16. Thomas R. Irmtrud J. Atlas de Ortopedia Maxilar: Diagnóstico. 1ra ed. Barcelona: Masson;1992.

17. Shruti K. Bhojraj N. Evaluation of changes in the dentoalveolar morphology in children with asthma. Pediatric Dental Journal 2012;22:95-102.

18. Susan H. Assessment of palatal height in children. Community Dent Oral Epidemiol 1981;9:44-47.

Recibido: 28/08/2019

Aceptado: 22/01/2020

Correspondencia: Daniel Maravillas García correo: marveldany@icloud.com 